\title{
Yield, Economic Benefit, Soil Water Balance, and Water Use Efficiency of Intercropped Maize/Potato in Responses to Mulching Practices on the Semiarid Loess Plateau
}

\author{
Junhong Xie ${ }^{1,2}$, Linlin Wang ${ }^{1,2}\left(\mathbb{D}\right.$, Lingling $\mathrm{Li}^{1,2, * \mathbb{D}}$, Sumera Anwar ${ }^{3}$, Zhuzhu Luo ${ }^{1,2}$, Effah Zechariah ${ }^{1,4}$ \\ and Setor Kwami Fudjoe ${ }^{1,2}$
}

1 State Key Laboratory of Aridland Crop Science, Gansu Agricultural University, Lanzhou 730070, China; xiejh@gsau.edu.cn (J.X.); wangll@gsau.edu.cn (L.W.); luozz@gsau.edu.cn (Z.L.); zechariah@st.gsau.edu.cn (E.Z.); setor@st.gsau.edu.cn (S.K.F.)

2 College of Agronomy, Gansu Agricultural University, Lanzhou 730070, China

3 Institute of Molecular Biology and Biotechnology, The University of Lahore, Lahore 54660, Pakistan; sumera.anwar@imbb.uol.edu.pk

4 College of Resources and Environment Science, Gansu Agricultural University, Lanzhou 730070, China

* Correspondence: lill@gsau.edu.cn

check for updates

Citation: Xie, J.; Wang, L.; Li, L.; Anwar, S.; Luo, Z.; Zechariah, E.; Kwami Fudjoe, S. Yield, Economic Benefit, Soil Water Balance, and Water Use Efficiency of Intercropped Maize/Potato in Responses to Mulching Practices on the Semiarid Loess Plateau. Agriculture 2021, 11, 1100. https://doi.org/10.3390/ agriculture 11111100

Academic Editors: Stefano Tavoletti, Martin Weih, M. Inés Mínguez and Claudia Di Bene

Received: 9 September 2021 Accepted: 2 November 2021 Published: 4 November 2021

Publisher's Note: MDPI stays neutral with regard to jurisdictional claims in published maps and institutional affiliations.

Copyright: (C) 2021 by the authors. Licensee MDPI, Basel, Switzerland. This article is an open access article distributed under the terms and conditions of the Creative Commons Attribution (CC BY) license (https:// creativecommons.org/licenses/by/ $4.0 /)$.

\begin{abstract}
Increasing agricultural productivity without undermining further the integrity of the Earth's environmental systems such as soil water balance are important tasks to ensure food security for an increasing global population in rainfed agriculture. The impact of intercropping maize (Zea mays L.) with potato (Solanum tuberosum L.) on yield, land equivalent ratios (LER), water equivalent ratio (WER), water use, energy output, and net economic return were examined under seven planting systems: potato grown solely or intercropped on the flat field without mulching, maize grown solely or intercropped with potato on ridges or flat field with or without plastic film mulched. The three intercropping systems had 3-13\% less water use than the monocropping. Among the intercropped systems, flat field caused more depletion of soil water than ridged field for both years. Compared to monocultures, intercropping with plastic film mulching and ridging significantly increased LER and WER. Meanwhile, intercropping with mulching and ridging significantly increased net economic return and energy output by $8 \%$ and $24 \%$, respectively, when compared to monocropping. These results suggest that maize under plastic film mulched ridge-furrow plot intercropped with potato under flat plot without mulching increased energy output, net economic return, and water use efficiency without increasing soil water depletion, which could be an optimal intercropping system for the semiarid farmland on the western Loess Plateau.
\end{abstract}

Keywords: intercropping; mulching; soil water balance; yield; water use efficiency; net economic return

\section{Introduction}

In the coming decades, a crucial challenge for humanity will be meeting future food demands without undermining further the integrity of the Earth's environmental systems [1-4]. This challenge would be met by crop diversification and trade-offs between productivity and other ecosystem services [5-7].

The Loess Plateau in China is characterized by an extremely hilly loess landscape and harsh climatic conditions, including frequent spring drought and severe soil erosion [8-11]. The average annual precipitation ranges from 300 to $600 \mathrm{~mm}$ which is the major water resource for agricultural production under a rainfed farming system [8,12,13]; however, in most cases, the water resource is inefficiently used [12]. Some major issues for crop production on the Loess Plateau are (1) low water-use efficiency (WUE) i.e., the low economic yield caused by drought stress (limited and erratic rainfall), (2) low decomposition of organic matter which affects soil fertility, and (3) high evaporation reducing the amount of water in the soil for crop growth [13-15]. Therefore, crop yield could be increased by 
maximizing WUE which is a major challenge for dryland farming in the semiarid area on the Loess Plateau [12,16].

In the western areas of Loess Plateau, the dryland farming under traditional agricultural production is dominated by the sole cropping of spring wheat (Triticum aestivum L.) and potato (Solanum tuberosum L.) [17-19]. Since the application of plastic film mulching technology at the end of the 20th century, maize has been widely planted in this region $[20,21]$. With the widespread application of plastic film fully mulched ridge-furrow system (FMRF) in the last decade or so, the planting area and yield of maize have increased recently in this region [18,20,22]. Maize has become a newly dominant crop in the western areas of the Loess Plateau [18]. FMRF greatly increased yield and WUE of maize by optimizing growth conditions including decreased soil evaporation, increased soil temperature $[20,21]$, improved nutrient availability, suppressed arable weed growth, and reduced competition of weeds for water and nutrients [23-25]. However, in addition to increasing maize yield, applications of FMRF are also associated with some environmental concerns, such as increasing greenhouse gas emission [26,27] and plastic-film residue pollution [28,29]. Also, high maize yield under FMRF has led to the depletion of soil water, thereby reducing soil water sustainability $[18,30]$.

Potato is an important food crop and is widely planted without plastic film mulching in this region; however, potato continuous cropping caused the severe depletion of soil water by high-water consumption [31] and soil-borne diseases, which restrains the potato yield [32]. A challenge for sustainable production of maize and potato in this region is to lessen the depletion of soil water while reducing the amounts of plastic film without harmful trade-offs between productivity and other ecosystem services.

Intercropping is a farming practice involving two or more crop species, or genotypes, growing together and coexisting for a time $[33,34]$. Intercropping increases crop yield $[7,35,36]$ and enhance resource use efficiencies such as land, light, water, and nutrients [5,37,38], and also provides numerous ecological benefits [1,39,40]. Some studies found that adding a species into an ecosystem would inevitably increase water consumption when the water resources are limited [40,41]. Because of this view, intercropping is rarely practiced on the western Loess Plateau. However, other studies demonstrated that the appropriate combination of crop species and planting density does not significantly increase the water use by intercropping [38,42-44]. The appropriate crop components, planting time, and planting patterns could make full use of the spatial structure of crops and enhance the crop utilization of agricultural resources and yield in intercropping [1,39]. Therefore, detailed studies are required to explore the effects of intercropping maize with potato on yield and WUE for maize grown under limited and erratic rainfall conditions with different plastic film mulching practices. Also, most of the studies examined the intercropping advantage in respective yield and WUE of component crops based on the relative density of each species, while little attention has been paid to the effects of intercropping on energy output and net economic return of the whole intercropping system. Therefore, the aims of this study were (i) to determine the effect of intercropping maize with potato on economic yield, water use, soil water balance, and WUE, and (ii) to evaluate the energy output and net economic return in the intercropping for selecting optimized intercropping pattern in the western areas of Loess Plateau.

\section{Materials and Methods}

\subsection{Experimental Site}

A field experiment was conducted at the Rainfed Agricultural Experimental station of Gansu Agricultural University on the western Loess Plateau ( $\left.35^{\circ} 28^{\prime} \mathrm{N}, 104^{\circ} 44^{\prime} \mathrm{E}\right)$ in Gansu Province, China during the 2014-2015 growing seasons. The long-term average annual rainfall of this area is $390 \mathrm{~mm}$, with about $54 \%$ occurring from July to September each year. The experimental site has a medium-temperate semiarid climate, annual mean air temperature of $6.4^{\circ} \mathrm{C}$. The mean frost-free period is 140 days and an annual watersurface evaporation demand of $1531 \mathrm{~mm}$. The Aeolian soil at the experimental site, known 
as Huangmian soil [45], is a calcaric cambisol (FAO Soil Classification). The field water holding capacity is about $0.256 \mathrm{~cm}^{3} \mathrm{~cm}^{-3}$ and the wilting point is $0.085 \mathrm{~cm}^{3} \mathrm{~cm}^{-3}$. The $\mathrm{pH}$ value of the field soil sampled from $0-20 \mathrm{~cm}$ depth $\left(0.01 \mathrm{M} \mathrm{CaCl}_{2}\right)$, is 8.4 . Soil samples were randomly collected at five points at the experimental site in April 2014 with the following characteristics: soil total nitrogen $(\mathrm{N})\left(1.1 \mathrm{~g} \cdot \mathrm{kg}^{-1}\right)$, organic matter content $\left(8.83 \mathrm{~g} \cdot \mathrm{kg}^{-1}\right)$, available phosphorus $\left(17 \mathrm{mg} \cdot \mathrm{kg}^{-1}\right)$, available potassium $\left(141 \mathrm{mg} \cdot \mathrm{kg}^{-1}\right)$ and bulk density at $0-20 \mathrm{~cm}$ depth $\left(1.18 \mathrm{~g} \cdot \mathrm{cm}^{-3}\right)$.

\subsection{Experimental Design}

The experiment was a randomized complete block design with seven cropping systems and three replications. Each plot was $6.6 \mathrm{~m}$ width $\times 10 \mathrm{~m}$ length. The width of each maize strip (including two rows of maize) or potato strip (including three rows of potato) in the intercropping system was $110 \mathrm{~cm}$; three maize strips and three potato strips were arranged in each intercropping plot $(1.1 \mathrm{~m} \times 3+1.1 \mathrm{~m} \times 3=6.6 \mathrm{~m})$. Seven cropping systems (treatments) were used in this study for both 2014 and 2015 growth seasons: (1) maize monocrop under plastic film fully mulched ridge-furrow plot (FMRFm), (2) maize monocrop under plastic film fully mulched flat plot (FMFm), (3) maize monocrop under flat plot without plastic film mulch (NMFm), (4) potato monocrop under flat plot without plastic film mulch (NMFp), (5) intercropping maize under plastic film fully mulched ridge-furrow plot with potato under flat plot without plastic film mulch (FMRFm/NMFp), (6) intercropping maize under plastic film fully mulched flat plot with potato under flat plot without plastic film mulch (FMFm/NMFp), and (7) intercropping maize under flat plot without plastic film mulch with potato under flat plot without plastic film mulch (NMFm/NMFp) (Figure 1). In order to investigate the water balance of intercropping systems, plots with different cropping systems were located in the same location for the two growing seasons, and the crops were grown and arranged in the same pattern for the two growing seasons.



Figure 1. Diagram of the different maize-potato intercropping systems at the Rainfed Agricultural Experiment Station of Gansu Agricultural University, Dingxi City ( $35^{\circ} 28^{\prime}$ N, $104^{\circ} 44^{\prime}$ E), Gansu Province, China. FMRF, film mulched ridge-furrow plot; FMF, film mulched flat plot; NMF, no mulching flat plot; $\mathrm{m}$, maize; $\mathrm{p}$, potato.

Intercropping system contained alternating two rows of maize and three rows of potato (Figure 1). For monocropping, the plant spacing in each row was $35 \mathrm{~cm}$ for maize at a density of 52,500 plants $\mathrm{ha}^{-1}$, and $53 \mathrm{~cm}$ for potato $\left(52,500\right.$ plants ha $\left.{ }^{-1}\right)$. Local maize 
variety (cv.) Funong 821 and potato cv. Longshu10 was sown by hand in late April and was harvested in early October for both years. All maize and potato residues were removed from the field after harvest, and plastic film was left to cover the soil until the following spring.

\subsection{Sampling and Measurements}

Soils from the surface to a depth of $200 \mathrm{~cm}$ were sampled at sowing (late April) and physiological maturity (early October) of maize and potato per year for measurement of water use (WU) and water use efficiency (WUE). For monoculture plots, three soil samples were randomly collected in the central rows of each plot at intervals of $10 \mathrm{~cm}$ within the first $50 \mathrm{~cm}$ depth and $30 \mathrm{~cm}$ intervals from $50 \mathrm{~cm}$ to $200 \mathrm{~cm}$. For intercropping plots, three soil samples were randomly collected in the central rows of the maize and potato strips. The fresh weight of the soil samples was determined immediately after sampling and the dry weight was determined after drying in a forced-air oven at $105^{\circ} \mathrm{C}$ until constant weight to calculate the soil moisture. Water use was calculated by the soil water balance equation $[13,18]$.

At maturity, all maize and potato plants in each plot were manually harvested. After harvest, the air-dried grains of maize were weighed for each plot to calculate grain yield and determine 100-grain weight. Fresh tuber yield of potato was recorded for each plot to measure the yield of potato. Additional five maize plants from each plot were sampled to determine ear number per plant, ear length $(\mathrm{cm})$, rows per ear, and grain number per row, and five potato plants from each plot were sampled to determine tuber number per plant, yield per plant, and commodity rate (\%). Land equivalent ratio (LER) was used to assess land productivity in intercropping and was calculated following [37,38]. If LER is higher than 1.0, it indicates an advantage in intercropping [46].

$$
\mathrm{LER}=(\mathrm{Yi}, \mathrm{x}) /(\mathrm{Ym}, \mathrm{x})+(\mathrm{Yi}, \mathrm{y}) /(\mathrm{Ym}, \mathrm{y})
$$

where $Y i, x$ and $Y i, y$ are the yield of maize and potato in intercrop, $Y m, x$ and $Y m, y$ are the yield of maize and potato in monocrops.

Water equivalent ratio (WER) was used to assess WUE in intercropping compared to monocropping $[37,38]$. If WER is lower than 1.0, it indicates water use disadvantage in intercropping.

$$
\text { WER }=\text { LERx }(\text { WUm,x }) / W U i+\text { LERy }(W U m, y) / W U i
$$

where LERx and LERy are the land productivity of maize and potato in monocropping, WUi is the actual water use of the whole intercropping system, and WUm,x and WUm,y are the actual water use of maize and potato in monocropping.

$\triangle W U$ was used to evaluate whether intercropping uses more or less water than monocultures [37], If $\Delta \mathrm{WU}$ is higher than 0 , intercropping consumes greater water than expected from the crop yields obtained.

$$
\Delta \mathrm{WU}=1 / \mathrm{WER}-1
$$

$\Delta \mathrm{Y}, \Delta \mathrm{E}$, and $\triangle \mathrm{NER}$ were calculated to express the relative changes in yield, energy output, and net economic return in intercropping compared to expectation for each species considering the relative density of maize and potato in monocultures, respectively $[37,38]$.

$$
\begin{gathered}
\Delta \mathrm{Y}=\frac{\mathrm{Yi}, \mathrm{a}-0.5 \times \mathrm{Ym}, \mathrm{a}}{\mathrm{Pi} \times \mathrm{Ym}, \mathrm{a}} \\
\Delta \mathrm{E}=\frac{\mathrm{Ei}-0.5 \times(\mathrm{Em}, \mathrm{x}+\mathrm{Em}, \mathrm{y})}{0.5 \times(\mathrm{Em}, \mathrm{x}+\mathrm{Em}, \mathrm{y})} \\
\Delta \mathrm{NER}=\frac{\mathrm{NERi}-0.5 \times(\mathrm{NERm}, \mathrm{x}+\mathrm{NERm}, \mathrm{y})}{0.5 \times(\mathrm{NERm}, \mathrm{x}+\mathrm{NERm}, \mathrm{y})}
\end{gathered}
$$


where 0.5 is the relative density of maize and potato in the intercrop, $\mathrm{Yi}$,a and $\mathrm{Ym}, \mathrm{a}$ are the yields of crop a in intercrop and monocrop, respectively, Ei is the energy output in the intercropping, $\mathrm{Em}, \mathrm{x}$ and $\mathrm{Em}, \mathrm{y}$ are the energy output of maize and potato in monocrop, NERt is the net economic return in the intercropping, NERm, $x$ and NERm, $y$ are the net economic return of maize and potato in monocrop.

Water use efficiency for economic yield per species (WUEy, $\mathrm{kg} \cdot \mathrm{m}^{-3}$ ) was calculated following [37].

$$
\mathrm{WUEy}=\mathrm{Yx} / \mathrm{WU}
$$

where $\mathrm{YX}$ is the yields of crop $\mathrm{X}$ in intercrop and monocrop $\left(\mathrm{kg} \cdot \mathrm{ha}^{-1}\right)$, WU is actual water use in the intercropping (expressed as the total water use in intercropping) or monocrop $\left(\mathrm{m}^{3} \cdot \mathrm{ha}^{-1}\right)$.

Energy output from the production was calculated by multiplying the amount of production and its corresponding energy equivalent, and the energy equivalent value used in this study is $3.8 \mathrm{MJ} \cdot \mathrm{kg}^{-1}$ for potato and $16.3 \mathrm{MJ} \cdot \mathrm{kg}^{-1}$ for maize, respectively [27]. Net economic return was calculated by the difference between the revenue of the product and the total production cost associated with agricultural inputs [18,47]. The average prices of maize, potato, and fertilizers are shown in detail in previous studies [18,27].

\subsection{Statistical Analysis}

All data were statistically analyzed for analysis of variance (ANOVA) as a completely randomized design with three replications using SAS software (SAS Institute, Inc., Cary, NC, USA). The significant differences among means were determined using Fisher's LSD test at $p \leq 0.05$.

\section{Results}

\subsection{Weather Conditions}

There was a large variation in the rainfall pattern during the two experimental years (Figure 2). The annual rainfall in $2014(389 \mathrm{~mm})$ was almost equal to the long-term average from 1970 to 2011 (390 mm), but it was lower in 2015 (335 mm). The rainfall during the growing season of maize in both $2014(285 \mathrm{~mm})$ and $2015(278 \mathrm{~mm})$ was less than the long-term average $(333 \mathrm{~mm})$ [18].

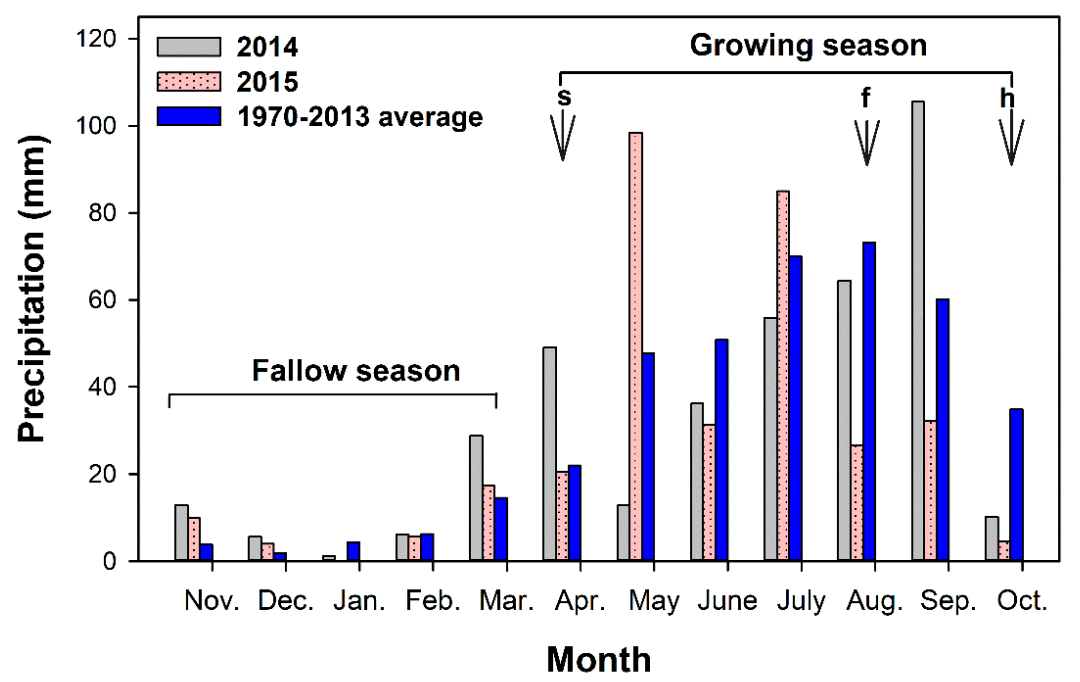

Figure 2. Average monthly precipitation in 2014 and 2015, and the long-term (1970-2013) monthly precipitation means at the Rainfed Agricultural Experiment Station of Gansu Agricultural University, Dingxi City $\left(35^{\circ} 28^{\prime} \mathrm{N}, 104^{\circ} 44^{\prime} \mathrm{E}\right)$, Gansu Province, China. The three major growth stages of maize, sowing (s), flowering (f), and harvest (h) are indicated on the graph. 


\subsection{Yield, Yield Components, and Land Equivalent Ratio}

Yield and yield components of maize and yield of potato were significantly affected by the planting system (Tables 1 and 2). The changing trend of yield and yield components of maize and potato in the two growing seasons were almost similar among the intercropping treatments; however, as a result of the severe drought, the potato yield was significantly lower in 2014 than that in 2015. On average, the FMRFm/NMFp treatment greatly increased ear number per plant, 100-grain weight, and yield per plant in maize compared to all other treatments. For maize, intercropping slightly increased ear number per plant and 100-grain weight, thereby increasing maize yield per plant with the highest maize yield achieved under FMRFm/NMFp (Table 1). Similarly, the potato yield was also improved by intercropping especially under FMRFm/NMFp. The intercropping increased the tuber number per plant resulting in high potato yield. The $\mathrm{F} \times \mathrm{W}$ interaction was statistically significant for potato yield per plant and commodity rate (Table 1$)$.

Maize yield in monocrop varied from a minimum of $6.4 \mathrm{Mg} \cdot \mathrm{ha}^{-1}$ under NMFm to a maximum of $10.4 \mathrm{Mg} \cdot \mathrm{ha}^{-1}$ under FMRFm (Table 2). Over the two growing seasons, the yield of maize under FMRFm and FMFm was 60 and 52\% higher than under NMFm, respectively. On average, maize yield in intercropping $\left(4.5 \cdot \mathrm{Mg} \mathrm{ha}^{-1}\right)$ was slightly greater than $50 \%$ of that in monocrop $\left(8.8 \cdot \mathrm{Mg} \mathrm{ha}^{-1}\right)$. Potato yield in monocrop also varied substantially from a minimum of $6.4 \mathrm{Mg} \cdot \mathrm{ha}^{-1}$ in 2015 to a maximum of $10.4 \mathrm{Mg} \cdot \mathrm{ha}^{-1}$ in 2014 . On average, potato yield in intercropping $\left(6.0 \mathrm{Mg} \cdot \mathrm{ha}^{-1}\right)$ was also slightly greater than $50 \%$ of that in monoculture (11.1 Mg.ha $\left.{ }^{-1}\right)$. For maize and potato, the greatest yield increase in intercropping was found under treatment of FMRFm/NMFp. Overall, the potato had a competitive advantage in the intercropping system over maize. The $\mathrm{F} \times \mathrm{W}$ interaction was statistically significant for potato yield and $\Delta Y$; however, the $C \times F$ interaction was not statistically significant for maize yield and $\Delta Y$ (Table 2). The planting system significantly affected the land equivalent ratio (LER) of the intercrop system (Table 3). The greatest LER was found under FMRFm/NMFp, followed by FMFm/NMFp, and NMFm/NMFp. The growing season in 2015 had greater partial LER than in 2014, suggesting that intercropping system enabled maize and potato to increase yield more efficiently in a relatively drier year. On average, LER was 1.11, 1.01, and 1.03 under FMRFm/NMFp, FMFm/NMFp, and NMFm/NMFp, respectively, showing a major increase in relative LER by intercropping. The changes in LER of maize, LER of potato, and LER of intercropping were not significant $(p>0.05)$. The $\mathrm{C} \times \mathrm{F}$ interaction was not statistically significant for LER of maize, LER of potato, and LER of intercropping (Table 3).

\subsection{Soil Water Storage, Water Use, and Water Balance}

Soil water storage, water use, and water balance varied between growing seasons and among cropping systems (Tables 4-6). Soil water storage was greater in 2014 than in 2015 and the depletion of soil water (i.e., soil water balance) during the growing season was less in 2014 than in 2015 (Table 4). On average, the intercropping had greater soil water storage at both sowing and harvest except for NMFp at sowing and FMFm/NMFp at harvest than monocrop. The depletion of soil water during the growing season was 12-26 mm less under FMRFm/NMFp and FMRFm, but it was greater by 3-36 $\mathrm{mm}$ in treatments of FMFm/NMFp, NMFm/NMFp, and NMFp than in FMFm and NMFm. The $\mathrm{C} \times \mathrm{F}$ interaction was not statistically significant for soil water balance (Table 4). 
Table 1. Average grain yield and yield components of maize, tuber number, yield, and commodity rate of potato under different planting systems during $2014-2015$.

\begin{tabular}{|c|c|c|c|c|c|c|c|c|c|c|}
\hline \multirow[b]{2}{*}{ Years } & \multirow[b]{2}{*}{$\begin{array}{l}\text { Cropping } \\
\text { System a }^{a}\end{array}$} & \multicolumn{6}{|c|}{ Maize Yield Components } & \multicolumn{3}{|c|}{ Potato Yield Components } \\
\hline & & $\begin{array}{c}\text { Ear Number } \\
\text { per Plant }\end{array}$ & $\begin{array}{l}\text { Ear Length } \\
\quad \text { (cm) }\end{array}$ & Rows per Ear & $\begin{array}{c}\text { Grain } \\
\text { Number per } \\
\text { Row }\end{array}$ & $\begin{array}{l}\text { 100-Grain } \\
\text { Weight (g) }\end{array}$ & $\begin{array}{c}\text { Yield } \\
\left.\text { (g plant }^{-1}\right)\end{array}$ & $\begin{array}{c}\text { Tuber } \\
\text { Number per } \\
\text { Plant }\end{array}$ & $\begin{array}{c}\text { Yield } \\
\left.\text { (g plant }^{-1}\right)\end{array}$ & $\begin{array}{l}\text { Commodity } \\
\text { Rate (\%) }\end{array}$ \\
\hline \multirow[t]{7}{*}{2014} & FMRFm/NMFp & $1.94 \mathrm{a}^{\mathrm{b}}$ & $21.5 \mathrm{a}$ & $14.9 \mathrm{a}$ & $36.5 \mathrm{a}$ & $58.1 \mathrm{a}$ & $218.2 \mathrm{a}$ & $6.1 \mathrm{a}$ & $473.5 \mathrm{a}$ & $78.6 \mathrm{a}$ \\
\hline & FMFm/NMFp & $1.77 \mathrm{~b}$ & $22.2 \mathrm{a}$ & $15.3 \mathrm{a}$ & $35.5 \mathrm{a}$ & $52.8 \mathrm{ab}$ & $192.8 \mathrm{~b}$ & $5.8 \mathrm{ab}$ & $467.7 \mathrm{ab}$ & $74.0 \mathrm{a}$ \\
\hline & NMFm/NMFp & $1.59 \mathrm{~b}$ & $22.1 \mathrm{a}$ & $15.3 \mathrm{a}$ & $36.5 \mathrm{a}$ & $55.4 \mathrm{ab}$ & $131.5 \mathrm{c}$ & $5.2 \mathrm{~b}$ & $434.5 \mathrm{~b}$ & $73.7 \mathrm{a}$ \\
\hline & FMRFm & $1.66 \mathrm{~b}$ & $22.8 \mathrm{a}$ & $15.1 \mathrm{a}$ & $37.3 \mathrm{a}$ & $53.9 \mathrm{ab}$ & $205.2 \mathrm{ab}$ & - & - & - \\
\hline & FMFm & $1.66 \mathrm{~b}$ & $22.1 \mathrm{a}$ & $15.5 \mathrm{a}$ & $36.1 \mathrm{a}$ & 56.7 a & $182.2 \mathrm{~b}$ & - & - & - \\
\hline & NMFm & $1.51 \mathrm{~b}$ & $21.6 \mathrm{a}$ & $15.0 \mathrm{a}$ & $33.9 \mathrm{a}$ & $52.4 \mathrm{~b}$ & $127.7 \mathrm{c}$ & - & - & - \\
\hline & NMFp & - & - & - & - & - & - & $5.3 \mathrm{~b}$ & $421.6 \mathrm{~b}$ & $74.1 \mathrm{a}$ \\
\hline \multirow[t]{7}{*}{2015} & FMRFm/NMFp & $1.50 \mathrm{a}$ & $19.9 \mathrm{a}$ & $13.6 \mathrm{a}$ & $32.8 \mathrm{a}$ & $52.3 \mathrm{a}$ & $197.2 \mathrm{a}$ & $3.3 \mathrm{a}$ & $228.1 \mathrm{a}$ & $25.8 \mathrm{a}$ \\
\hline & FMFm/NMFp & $1.40 \mathrm{~b}$ & $19.8 \mathrm{a}$ & $13.7 \mathrm{a}$ & $31.9 \mathrm{a}$ & $49.6 \mathrm{ab}$ & $174.2 \mathrm{~b}$ & $3.0 \mathrm{ab}$ & $222.9 \mathrm{ab}$ & $23.8 \mathrm{ab}$ \\
\hline & NMFm/NMFp & $1.21 \mathrm{bc}$ & $19.8 \mathrm{a}$ & $13.7 \mathrm{a}$ & $32.8 \mathrm{a}$ & $49.9 \mathrm{ab}$ & $118.7 \mathrm{c}$ & $2.8 \mathrm{~b}$ & $202.9 \mathrm{~b}$ & $20.5 \mathrm{ab}$ \\
\hline & FMRFm & $1.28 \mathrm{~b}$ & $20.4 \mathrm{a}$ & $13.5 \mathrm{a}$ & $33.5 \mathrm{a}$ & $48.5 \mathrm{ab}$ & $185.4 \mathrm{ab}$ & - & - & - \\
\hline & FMFm & $1.28 \mathrm{~b}$ & $19.7 \mathrm{a}$ & $13.8 \mathrm{a}$ & $32.5 \mathrm{a}$ & $51.1 \mathrm{ab}$ & $164.6 \mathrm{~b}$ & - & - & - \\
\hline & NMFm & $1.17 \mathrm{c}$ & $18.9 \mathrm{a}$ & $13.2 \mathrm{a}$ & $30.5 \mathrm{a}$ & $47.2 \mathrm{~b}$ & $115.3 \mathrm{c}$ & - & - & - \\
\hline & NMFp & - & - & - & - & - & - & $2.7 \mathrm{~b}$ & $181.3 \mathrm{c}$ & $18.3 \mathrm{~b}$ \\
\hline \multirow{2}{*}{ Year $(\mathrm{Y})$} & & $1.30 \mathrm{~b}$ & $19.8 \mathrm{a}$ & $13.6 \mathrm{~b}$ & $32.3 \mathrm{~b}$ & $49.8 \mathrm{~b}$ & $159.2 \mathrm{~b}$ & $3.0 \mathrm{~b}$ & $258.8 \mathrm{~b}$ & $34.3 \mathrm{~b}$ \\
\hline & FMRFm/NMFp & $1.72 \mathrm{a}$ & $20.7 \mathrm{a}$ & $14.3 \mathrm{a}$ & $34.6 \mathrm{ab}$ & $55.2 \mathrm{a}$ & $207.7 \mathrm{a}$ & $4.7 \mathrm{a}$ & $350.8 \mathrm{a}$ & $52.2 \mathrm{a}$ \\
\hline \multirow{6}{*}{$\begin{array}{l}\text { Cropping } \\
\text { system (C) }\end{array}$} & FMFm/NMFp & $1.58 \mathrm{~b}$ & $21.0 \mathrm{a}$ & $14.5 \mathrm{a}$ & $33.7 \mathrm{ab}$ & $51.2 \mathrm{ab}$ & $183.5 \mathrm{~b}$ & $4.4 \mathrm{ab}$ & $345.3 \mathrm{ab}$ & $48.9 \mathrm{ab}$ \\
\hline & NMFm/NMFp & $1.40 \mathrm{bc}$ & $21.0 \mathrm{a}$ & $14.5 \mathrm{a}$ & $34.6 \mathrm{ab}$ & $52.6 \mathrm{ab}$ & $125.1 \mathrm{c}$ & $4.0 \mathrm{~b}$ & $318.7 \mathrm{~b}$ & $47.1 \mathrm{~b}$ \\
\hline & FMRFm & $1.47 \mathrm{~b}$ & $21.6 \mathrm{a}$ & $14.3 \mathrm{a}$ & $35.4 \mathrm{a}$ & $51.2 \mathrm{ab}$ & $195.3 \mathrm{ab}$ & - & - & - \\
\hline & FMFm & $1.47 \mathrm{~b}$ & $20.9 a$ & $14.6 \mathrm{a}$ & $34.3 \mathrm{ab}$ & $53.9 \mathrm{ab}$ & $173.4 \mathrm{~b}$ & - & - & - \\
\hline & NMFm & $1.34 \mathrm{c}$ & $20.3 a$ & $14.1 \mathrm{a}$ & $32.2 \mathrm{~b}$ & $49.8 \mathrm{~b}$ & $121.5 \mathrm{c}$ & - & - & - \\
\hline & NMFp & - & - & - & - & - & - & $4.0 \mathrm{~b}$ & $301.5 \mathrm{c}$ & $46.2 \mathrm{~b}$ \\
\hline $\mathrm{Y}$ & & * & ns & $*$ & * & * & * & $* * *$ & $* * *$ & $* * *$ \\
\hline $\mathrm{C}$ & & $* *$ & ns & ns & * & * & $* *$ & * & * & * \\
\hline $\mathrm{Y} \times \mathrm{C}$ & & ns & ns & ns & ns & ns & ns & ns & * & * \\
\hline
\end{tabular}

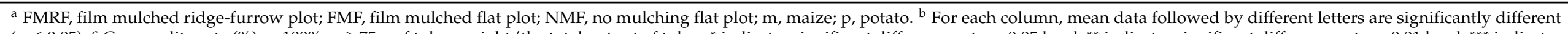

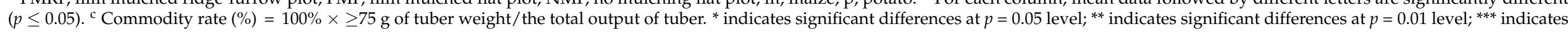
significant differences at $p=0.001$ level; ns: not significant. 
Table 2. Yields of maize and potato crops in the intercropping compared with monocrop in 2014-2015.

\begin{tabular}{|c|c|c|c|c|c|c|c|}
\hline \multirow{2}{*}{ Years } & \multirow{2}{*}{$\begin{array}{c}\text { Mulch Practices } \\
\text { of Maize }\end{array}$} & \multicolumn{3}{|c|}{ Maize Yield $\left(\mathrm{Mg} \mathrm{ha}^{-1}\right)^{b}$} & \multicolumn{3}{|c|}{ Potato Yield (Mg ha $\left.{ }^{-1}\right)$} \\
\hline & & Intercrop & Monocrop & $\Delta Y(\%)$ & Intercrop & Monocrop & $\Delta \mathrm{Y}(\%)$ \\
\hline \multirow[t]{3}{*}{2014} & FMRF & $5.45 \mathrm{a}^{\mathrm{c}}$ & $10.43 \mathrm{a}$ & 5 & $7.92 \mathrm{a}$ & 13.80 & $15^{\sharp}$ \\
\hline & FMF & $4.50 \mathrm{~b}$ & $9.78 \mathrm{a}$ & $-8 *$ & $6.77 \mathrm{~b}$ & 13.80 & -2 \\
\hline & NMF & $3.25 c$ & $6.35 \mathrm{~b}$ & 2 & $7.22 \mathrm{~b}$ & 13.80 & 5 \\
\hline \multirow{3}{*}{2015} & FMRF & $5.45 \mathrm{a}$ & $10.07 \mathrm{a}$ & $8^{*}$ & $4.90 \mathrm{a}$ & 8.46 & $16^{\sharp}$ \\
\hline & FMF & $5.13 \mathrm{a}$ & $9.56 \mathrm{a}$ & 7 & $4.66 \mathrm{a}$ & 8.46 & $10^{\sharp}$ \\
\hline & NMF & $3.32 \mathrm{~b}$ & $6.40 \mathrm{~b}$ & 4 & $4.24 \mathrm{~b}$ & 8.46 & 0 \\
\hline \multicolumn{8}{|l|}{ Average } \\
\hline \multirow[t]{2}{*}{ Year $(\mathrm{Y})$} & 2014 & $4.40 \mathrm{a}$ & $8.85 \mathrm{a}$ & 0 & $7.30 \mathrm{a}$ & $13.80 \mathrm{a}$ & 6 \\
\hline & 2015 & $4.63 \mathrm{a}$ & $8.68 \mathrm{a}$ & 6 & $4.60 \mathrm{~b}$ & $8.46 \mathrm{~b}$ & $9 \sharp$ \\
\hline \multirow{3}{*}{$\begin{array}{l}\text { Cropping } \\
\text { system } \\
\text { (C) }\end{array}$} & FMRF & $5.45 \mathrm{a}$ & $10.25 \mathrm{a}$ & 6 & $6.41 \mathrm{a}$ & 11.13 & $15^{\sharp}$ \\
\hline & FMF & $4.82 \mathrm{~b}$ & $9.67 \mathrm{a}$ & 0 & $5.72 \mathrm{~b}$ & 11.13 & 3 \\
\hline & NMF & $3.28 \mathrm{c}$ & $6.38 \mathrm{~b}$ & 3 & $5.73 \mathrm{~b}$ & 11.13 & 3 \\
\hline $\mathrm{Y}$ & & ns & ns & ns & $* * *$ & $* * *$ & $* *$ \\
\hline $\mathrm{C}$ & & $* *$ & $*$ & ns & $*$ & - & * \\
\hline $\mathrm{Y} \times \mathrm{C}$ & & ns & ns & ns & * & - & * \\
\hline
\end{tabular}

${ }^{a}$ FMRF, film mulched ridge-furrow plot; FMF, film mulched flat plot; NMF, no mulching flat plot. ${ }^{\mathrm{b}} \mathrm{Mg}$ ha ${ }^{-1}$, tons per hectare; $\Delta \mathrm{Y}$ indicates yield increase taking into account the relative density of maize and potato in the intercrop ( $\mathrm{Pi}=0.5$ for both component crops), which is calculated as $\left(\mathrm{Y}_{\text {int }}-0.5 \times \mathrm{Y}_{\text {mono }}\right) /\left(0.5 \times \mathrm{Y}_{\text {mono }}\right)$. ${ }^{c}$ Mean data of each column for the same year followed by different small letters are significantly different $(p \leq 0.05)$. $\sharp$ Indicates significant differences among treatments $(p \leq 0.05) .{ }^{*}$ indicates significant differences at $p=0.05$ level; ${ }^{* *}$ indicates significant differences at $p=0.01$ level; ${ }^{* * *}$ indicates significant differences at $p=0.001$ level; ns: not significant.

Table 3. Land equivalent ratio in the maize/potato intercropping system in 2014-2015.

\begin{tabular}{|c|c|c|c|c|}
\hline Years & $\begin{array}{c}\text { Mulch Practices of } \\
\text { Maize }^{\text {a }}\end{array}$ & LER Maize & LER Potato & LER Intercropping \\
\hline \multirow[t]{3}{*}{2014} & FMRF & $0.52 \mathrm{a}^{\mathrm{b}}$ & $0.57 \mathrm{a}$ & $1.09 \mathrm{a}$ \\
\hline & FMF & $0.46 \mathrm{~b}$ & $0.49 \mathrm{~b}$ & $0.95 \mathrm{~b}$ \\
\hline & NMF & $0.51 \mathrm{ab}$ & $0.52 \mathrm{ab}$ & $1.03 \mathrm{ab}$ \\
\hline \multirow[t]{3}{*}{2015} & FMRF & $0.54 \mathrm{a}$ & $0.58 \mathrm{a}$ & $1.12 \mathrm{a}$ \\
\hline & FMF & $0.54 \mathrm{a}$ & $0.55 \mathrm{ab}$ & $1.09 \mathrm{ab}$ \\
\hline & NMF & $0.52 \mathrm{a}$ & $0.50 \mathrm{~b}$ & $1.02 \mathrm{~b}$ \\
\hline \multicolumn{5}{|l|}{ Average } \\
\hline \multirow{5}{*}{ Cropping system (C) } & 2014 & $0.50 \mathrm{a}$ & $0.53 \mathrm{a}$ & $1.03 \mathrm{a}$ \\
\hline & 2015 & $0.53 \mathrm{a}$ & $0.54 \mathrm{a}$ & $1.08 \mathrm{a}$ \\
\hline & FMRF & $0.53 \mathrm{a}$ & $0.58 \mathrm{a}$ & $1.11 \mathrm{a}$ \\
\hline & FMF & $0.50 \mathrm{a}$ & $0.51 \mathrm{~b}$ & $1.01 \mathrm{~b}$ \\
\hline & NMF & $0.52 \mathrm{a}$ & $0.51 \mathrm{~b}$ & $1.03 \mathrm{~b}$ \\
\hline $\mathrm{Y}$ & & ns & ns & ns \\
\hline $\mathrm{C}$ & & ns & $*$ & $*$ \\
\hline $\mathrm{Y} \times \mathrm{C}$ & & ns & ns & ns \\
\hline
\end{tabular}

${ }^{a}$ FMRF, film mulched ridge-furrow plot; FMF, film mulched flat plot; NMF, no mulching flat plot; LER, land equivalent ratio. ${ }^{\mathrm{b}}$ Within a column for the same year, means followed by different small letters are significantly different $(p \leq 0.05) .{ }^{*}$ indicates significant differences at $p=0.05$ level; ns: not significant. 
Table 4. Soil water storage in the $0-2 \mathrm{~m}$ depth at sowing and harvesting and soil water balance during the growing season under different planting systems in 2014-2015.

\begin{tabular}{|c|c|c|c|c|}
\hline \multirow{2}{*}{ Years } & \multirow{2}{*}{ Cropping System ${ }^{a}$} & \multicolumn{2}{|c|}{ Soil Water Storage (mm) } & \multirow{2}{*}{$\begin{array}{c}\text { Soil Water Balance } \\
(\mathrm{mm})\end{array}$} \\
\hline & & Sowing & Harvesting & \\
\hline \multirow[t]{7}{*}{2014} & FMRFm/NMFp & $405 \mathrm{a}^{\mathrm{b}}$ & $388 \mathrm{a}$ & $-17 \mathrm{a}$ \\
\hline & FMFm/NMFp & $405 \mathrm{a}$ & $343 \mathrm{bc}$ & $-62 c$ \\
\hline & NMFm/NMFp & $405 \mathrm{a}$ & $367 \mathrm{ab}$ & $-38 b$ \\
\hline & FMRFm & $405 \mathrm{a}$ & 392 a & $-13 \mathrm{a}$ \\
\hline & FMFm & $405 \mathrm{a}$ & $371 \mathrm{ab}$ & $-34 b$ \\
\hline & NMFm & $405 \mathrm{a}$ & $344 \mathrm{bc}$ & $-61 c$ \\
\hline & NMFp & $405 a$ & $330 \mathrm{c}$ & $-75 \mathrm{~d}$ \\
\hline \multirow[t]{7}{*}{2015} & FMRFm/NMFp & $398 \mathrm{a}$ & 326 a & $-72 b$ \\
\hline & FMFm/NMFp & $400 \mathrm{a}$ & $301 \mathrm{ab}$ & $-99 c$ \\
\hline & NMFm/NMFp & $401 \mathrm{a}$ & $296 \mathrm{~b}$ & $-105 \mathrm{~cd}$ \\
\hline & FMRFm & $378 \mathrm{a}$ & $308 \mathrm{ab}$ & $-70 \mathrm{~b}$ \\
\hline & FMFm & $388 \mathrm{a}$ & $287 \mathrm{~b}$ & $-101 \mathrm{~cd}$ \\
\hline & NMFm & $336 \mathrm{~b}$ & $283 \mathrm{~b}$ & $-53 \mathrm{a}$ \\
\hline & NMFp & $400 \mathrm{a}$ & $291 b$ & $-109 \mathrm{~d}$ \\
\hline \multicolumn{5}{|l|}{ Average } \\
\hline \multirow{9}{*}{ Cropping system (C) } & 2014 & $405 \mathrm{a}$ & $362 \mathrm{a}$ & $-43 a$ \\
\hline & 2015 & $386 \mathrm{a}$ & $299 \mathrm{~b}$ & $-87 \mathrm{~b}$ \\
\hline & FMRFm/NMFp & $402 \mathrm{a}$ & $357 \mathrm{a}$ & $-45 a$ \\
\hline & FMFm/NMFp & $403 a$ & $322 \mathrm{bc}$ & $-81 \mathrm{~d}$ \\
\hline & NMFm/NMFp & $403 a$ & $332 \mathrm{abc}$ & $-71 c$ \\
\hline & FMRFm & $392 a b$ & $350 \mathrm{ab}$ & $-42 \mathrm{a}$ \\
\hline & FMFm & $397 \mathrm{ab}$ & $329 \mathrm{abc}$ & $-68 c$ \\
\hline & NMFm & $371 \mathrm{~b}$ & $314 \mathrm{c}$ & $-57 \mathrm{~b}$ \\
\hline & NMFp & $403 \mathrm{a}$ & $310 c$ & $-93 \mathrm{e}$ \\
\hline $\mathrm{Y}$ & & ns & * & * \\
\hline $\mathrm{C}$ & & * & * & $* *$ \\
\hline $\mathrm{Y} \times \mathrm{C}$ & & ns & ns & * \\
\hline
\end{tabular}

${ }^{a}$ FM: film mulching; RF: ridge-furrow; F: flat plot; m: maize; p: potato. ${ }^{\mathrm{b}}$ Within a column for the same year, means followed by different letters are significantly different $(p \leq 0.05) .{ }^{*}$ indicates significant differences at $p=0.05$ level; ${ }^{* *}$ indcated significant differences at $p=0.01$ level; ns: not significant.

Table 5. Average soil water storage and soil water storage deficit degree for the $0-50,50-110$, and $110-200 \mathrm{~cm}$ soil layers at the harvesting of the intercropping system compared with monocrop during 2014-2015.

\begin{tabular}{|c|c|c|c|c|c|c|c|}
\hline \multirow[b]{2}{*}{ Soil Layers } & \multirow{2}{*}{$\begin{array}{c}\text { Mulch } \\
\text { Practices of } \\
\text { Maize }^{\text {a }}\end{array}$} & \multicolumn{3}{|c|}{ Soil Water Storage (mm) } & \multicolumn{3}{|c|}{ Soil Water Storage Deficit Degree (\%) } \\
\hline & & Intercrop & $\begin{array}{c}\text { Maize } \\
\text { Monocrop }\end{array}$ & $\begin{array}{c}\text { Potato } \\
\text { Monocrop }\end{array}$ & Intercrop & $\begin{array}{c}\text { Maize } \\
\text { Monocrop }\end{array}$ & $\begin{array}{c}\text { Potato } \\
\text { Monocrop }\end{array}$ \\
\hline \multirow[t]{3}{*}{$0-50 \mathrm{~cm}$} & FMRF & $89 \mathrm{a}^{\mathrm{b}}$ & $77 \mathrm{a}$ & 54 & $30.6 \mathrm{~b}$ & $39.6 \mathrm{~b}$ & 57.8 \\
\hline & FMF & $71 \mathrm{~b}$ & $76 \mathrm{a}$ & 54 & $44.2 \mathrm{a}$ & $40.8 \mathrm{~b}$ & 57.8 \\
\hline & NMF & $70 \mathrm{~b}$ & $64 \mathrm{~b}$ & 54 & $45.1 \mathrm{a}$ & $50.1 \mathrm{a}$ & 57.8 \\
\hline \multirow[t]{3}{*}{$50-110 \mathrm{~cm}$} & FMRF & $90 \mathrm{a}$ & $85 \mathrm{a}$ & 68 & $50.1 \mathrm{a}$ & $54.1 \mathrm{~b}$ & 67.2 \\
\hline & FMF & $91 \mathrm{a}$ & $86 \mathrm{a}$ & 68 & $49.5 \mathrm{a}$ & $53.1 \mathrm{~b}$ & 67.2 \\
\hline & NMF & 88 a & $76 \mathrm{~b}$ & 68 & $51.3 \mathrm{a}$ & 60.9 a & 67.2 \\
\hline \multirow[t]{3}{*}{$110-200 \mathrm{~cm}$} & FMRF & $178 \mathrm{a}$ & $188 \mathrm{a}$ & 188 & $40.7 \mathrm{~b}$ & $33.1 \mathrm{c}$ & 33.1 \\
\hline & FMF & $160 \mathrm{~b}$ & $167 \mathrm{~b}$ & 188 & $55.1 \mathrm{a}$ & $49.4 \mathrm{~b}$ & 33.1 \\
\hline & NMF & $173 \mathrm{a}$ & $145 \mathrm{c}$ & 188 & $44.6 \mathrm{~b}$ & $66.9 \mathrm{a}$ & 33.1 \\
\hline
\end{tabular}

${ }^{a}$ FMRF, film mulched ridge-furrow plot; FMF, film mulched flat plot; NMF, no mulching flat plot; LER, land equivalent ratio. ${ }^{\mathrm{b}}$ Means followed by different letters are significantly different $(p \leq 0.05)$. 
Table 6. Water use (WU) of the intercropping system compared with monocrop in 2014-2015.

\begin{tabular}{|c|c|c|c|c|c|}
\hline \multirow{2}{*}{ Years } & \multirow{2}{*}{ Mulching Practices of Maize ${ }^{a}$} & \multicolumn{3}{|c|}{ Water Use (mm) } & \multirow{2}{*}{$\Delta W U(\%)^{b}$} \\
\hline & & Intercrop & Maize Monocrop & Potato Monocrop & \\
\hline \multirow[t]{3}{*}{2014} & FMRF & $329 \mathrm{a}^{\mathrm{c}}$ & $315 \mathrm{a}$ & 334 & $-7.57 c$ \\
\hline & FMF & 329 a & 342 a & 334 & $2.26 \mathrm{a}$ \\
\hline & NMF & $334 \mathrm{a}$ & $325 \mathrm{a}$ & 334 & $-1.98 b$ \\
\hline \multirow[t]{3}{*}{2015} & FMRF & $339 a$ & $350 \mathrm{a}$ & 383 & $-17.59 c^{*}$ \\
\hline & FMF & $346 \mathrm{a}$ & 349 a & 383 & $-12.99 b^{*}$ \\
\hline & NMF & 353 a & 331 a & 383 & $-2.84 \mathrm{a}$ \\
\hline \multicolumn{6}{|l|}{ Average } \\
\hline \multirow{2}{*}{ Year $(Y)$} & 2014 & $331 \mathrm{a}$ & $327 \mathrm{a}$ & $334 \mathrm{a}$ & $-2.43 \mathrm{a}$ \\
\hline & 2015 & $346 a$ & 343 a & $383 \mathrm{~b}$ & $-11.14 b^{\sharp}$ \\
\hline \multirow{3}{*}{$\begin{array}{l}\text { Cropping } \\
\text { system (C) }\end{array}$} & FMRF & 334 a & 332 a & 358 & $-12.82 \mathrm{a}^{\sharp}$ \\
\hline & FMF & $338 \mathrm{a}$ & 345 a & 358 & $-5.22 \mathrm{~b}$ \\
\hline & NMF & $344 \mathrm{a}$ & $328 \mathrm{a}$ & 358 & $-2.75 c$ \\
\hline $\mathrm{Y}$ & & ns & ns & ns & $* *$ \\
\hline $\mathrm{C}$ & & ns & ns & - & $* *$ \\
\hline $\mathrm{Y} \times \mathrm{C}$ & & ns & ns & - & * \\
\hline
\end{tabular}

${ }^{a}$ FMRF, film mulched ridge-furrow plot; FMF, film mulched flat plot; NMF, no mulching flat plot; ${ }^{\mathrm{b}} \Delta \mathrm{WU}$ : difference in water use between intercropping and monocrop. If $\Delta \mathrm{WU}$ is less than 0 , intercropping consumes less water than expected from the crop yields obtained. ${ }^{\mathrm{c}}$ The means followed by different letters are significantly different $(p \leq 0.05)$. $\sharp$ Indicates significant differences among treatments $(p \leq 0.05)$.

${ }^{*}$ indicates significant differences at $p=0.05$ level; ${ }^{* *}$ indicates significant differences at $p=0.01$ level; ns: not significant.

Intercropping reduced the soil water storage deficit degree in the $0-110 \mathrm{~cm}$ soil layer as compared to monoculture, especially for FMRFm/NMFp (Table 5). Compared to maize monocrop, potato monocrop enhanced water use and increased soil water storage deficit degree in the 0-110 cm soil layer; however, intercropping under FMRFm/NMFp and FMFm/NMFp enhanced the extraction of stored soil water in the 110-200 cm soil layers, thereby increasing soil water storage deficit degree in the deep soil layer. However, considering the relative density of maize and potato, $\triangle W U$ was less except for FMFm/NMFp in 2014 (Table 6). On average, $\Delta W U$ was $-12.8,-5.2$, and $-2.8 \%$ under FMRFm/NMFp, FMFm/NMFp, and NMFc/NMFp, respectively. Overall, the potato had significantly greater water use by $26 \mathrm{~mm}$ than maize (Table 6 ). The $\mathrm{C} \times \mathrm{F}$ interaction was not statistically significant for $\Delta \mathrm{WU}$ (Table 6).

\subsection{Water Use Efficiency}

Water use efficiency (WUEy) per species varied with growing season and planting system (Table 7). The WUEy of intercropped maize and potato (economic yield per unit of total system water extraction) was significantly lower than the monocrop. Considering that the intercrop had only $50 \%$ maize and $50 \%$ potato, WUEy of maize in intercropping was significantly higher under FMRFm/NMFp and FMFm/NMFp than under NMFm/NMFp. However, WUEy of potato in intercropping was greatly increased compared to potato monocrop except for FMFm/NMFp and NMFm/NMFp in 2014. On average, considering the relative density of maize in the intercropping, the greatest WUEy of maize in intercropping was found under FMRFm/NMFp, followed by FMFm/NMFp and NMFm/NMFp. Considering the relative density of maize, intercropping did not increase the WUEy of maize when compared to that in monocrop. Similarly, the greatest WUEy of intercropped potato was also found under FMRFm/NMFp, followed by FMFm/NMFp and NMFm/NMFp. However, when the relative density of potato in the intercropping was considered, the WUEy of potato in the intercropping was greatly increased. The partial WER of maize did not differ between years, but partial WER of potato was significantly greater in 2015 than in 2014, suggesting that the intercropping enabled potato use water more efficiently in a relatively drier year. 
Table 7. Water use efficiency for yield and water equivalent ratio in maize/potato intercropping system compared with monocrop in 2014-2015.

\begin{tabular}{|c|c|c|c|c|c|c|c|c|}
\hline \multirow{2}{*}{ Years } & \multirow{2}{*}{$\begin{array}{c}\text { Mulch } \\
\text { Practices of } \\
\text { Maize }^{\mathrm{a}}\end{array}$} & \multicolumn{2}{|c|}{ WUEy of Maize $\left(\mathrm{kg} \mathrm{m}^{-3}\right)$} & \multicolumn{2}{|c|}{ WUEy of Potato $\left(\mathrm{kg} \mathrm{m}^{-3}\right)$} & \multicolumn{3}{|c|}{ WER } \\
\hline & & Intercrop & Monocrop & Intercrop & Monocrop & Maize & Potato & Sum \\
\hline \multirow[t]{3}{*}{2014} & FMRF & $1.66 \mathrm{a}^{\mathrm{b}}$ & $3.32 \mathrm{a}$ & $2.41 \mathrm{a}$ & 4.13 & $0.50 \mathrm{a}$ & $0.58 \mathrm{a}$ & $1.08 \mathrm{a}^{\sharp}$ \\
\hline & FMF & $1.37 \mathrm{~b}$ & $2.86 \mathrm{~b}$ & $2.06 \mathrm{~b}$ & 4.13 & $0.48 \mathrm{a}$ & $0.50 \mathrm{~b}$ & $0.98 \mathrm{~b}$ \\
\hline & NMF & $0.97 \mathrm{c}$ & $1.95 \mathrm{c}$ & $2.16 \mathrm{ab}$ & 4.13 & $0.50 \mathrm{a}$ & $0.52 \mathrm{ab}$ & $1.02 \mathrm{ab}$ \\
\hline \multirow[t]{3}{*}{2015} & FMRF & $1.61 \mathrm{a}$ & $2.88 \mathrm{a}$ & 1.45 & 2.21 & $0.56 \mathrm{a}$ & $0.65 \mathrm{a}$ & $1.21 \mathrm{a}^{\sharp}$ \\
\hline & FMF & $1.48 \mathrm{a}$ & $2.74 \mathrm{a}$ & 1.35 & 2.21 & $0.54 \mathrm{a}$ & $0.61 \mathrm{ab}$ & $1.15 \mathrm{ab}$ \\
\hline & NMF & $0.94 \mathrm{~b}$ & $1.93 \mathrm{~b}$ & 1.20 & 2.21 & $0.49 \mathrm{a}$ & $0.54 \mathrm{~b}$ & $1.03 \mathrm{~b}$ \\
\hline \multirow{3}{*}{$\begin{array}{l}\text { Average } \\
\text { Years (Y) }\end{array}$} & & & & & & & & \\
\hline & 2014 & $1.33 \mathrm{a}$ & $2.71 \mathrm{a}$ & $2.21 \mathrm{a}$ & $4.13 \mathrm{a}$ & $0.49 \mathrm{a}$ & $0.53 \mathrm{~b}$ & $1.03 \mathrm{~b}$ \\
\hline & 2015 & $1.34 \mathrm{a}$ & $2.52 \mathrm{a}$ & $1.33 \mathrm{~b}$ & $2.21 \mathrm{~b}$ & $0.53 \mathrm{a}$ & $0.60 \mathrm{a}$ & $1.13 \mathrm{a}$ \\
\hline \multirow{3}{*}{$\begin{array}{l}\text { Cropping } \\
\text { system (C) }\end{array}$} & FMRF & $1.63 \mathrm{a}$ & $3.08 \mathrm{a}$ & $1.92 \mathrm{a}$ & 3.11 & $0.53 \mathrm{a}$ & $0.62 \mathrm{a}$ & $1.15 \mathrm{a}^{\sharp}$ \\
\hline & FMF & $1.43 \mathrm{a}$ & $2.80 \mathrm{a}$ & $1.69 \mathrm{~b}$ & 3.11 & $0.51 \mathrm{a}$ & $0.55 \mathrm{ab}$ & $1.06 \mathrm{~b}$ \\
\hline & NMF & $0.96 \mathrm{~b}$ & $1.94 \mathrm{~b}$ & $1.67 \mathrm{~b}$ & 3.11 & $0.49 \mathrm{a}$ & $0.54 \mathrm{~b}$ & $1.03 \mathrm{~b}$ \\
\hline Y & & ns & ns & $*$ & $*$ & ns & * & $*$ \\
\hline C & & $*$ & $*$ & $*$ & - & ns & $*$ & $* *$ \\
\hline $\mathrm{Y} \times \mathrm{C}$ & & ns & ns & ns & - & ns & ns & * \\
\hline
\end{tabular}

a FMRF, film mulched ridge-furrow plot; FMF, film mulched flat plot; NMF, no mulching flat plot; LER, land equivalent ratio; WUEg: water use efficiency for yield; WER: water equivalent ratio. ${ }^{b}$ Within a column for the same year, means followed by different letters are significantly different $(p \leq 0.05)$. $\sharp$ Indicates significant differences among treatments $(p \leq 0.05) .{ }^{*}$ indicates significant differences at $p=0.05$ level; ${ }^{* *}$ indicates significant differences at $p=0.01$ level; ns: not significant.

The water equivalent ratio (WER) was 1.03 and 1.13 in 2014 and 2015, respectively, which means that water productivity was substantially improved by intercropping, especially in the drier year (i.e., 2015) (Table 7). On average, the partial WER of potato was $0.62,0.55$, and 0.54 , while that of maize was similar to its sowing proportion of 0.5 , suggesting that intercropped potato has a greater water use advantage than intercropped maize. The greatest WER was found under FMRFm/NMFp, followed by FMFm/NMFp and NMFm/NMFp, indicating that FMRFm/NMFp achieved more water use advantage than other planting systems.

\subsection{Energy Output and Net Economic Return}

Maize monocrop in both 2014 and 2015 had greater energy output than its intercropping and potato monocrop (Table 8). Plastic film mulching significantly increased the energy output of maize in monocrop, especially under the ridge-furrow mulching system. Energy output for both intercropping and monocrop was not significantly different for maize between 2014 and 2015, but for potato monocrop, it was significantly more in 2014 than in 2015. On average, when the relative density of maize and potato was considered in the intercropping, the energy output under FMRFm/NMFp, FMFm/NMFp, and NMFm/NMFp was $8.1 \%(p<0.05), 0.3 \%(p>0.05)$, and $2.7 \%(p>0.05)$ greater than that under monocrop, respectively.

Overall, maize monocrop had a greater net economic return than intercropping and potato monocrop (Table 8). Compared to maize monocrop, intercropping increased net economic return except for FMFm/ NMFp in 2014, but reduced net economic return in 2015. The net economic return for intercropping and potato monocrop was significantly less in 2015 than in 2014, but for maize monocrop, it was not significantly different between 2014 and 2015. Similarly, intercropping increased net economic return more in 2015 than in 2014. On average, considering the relative density of maize and potato in intercropping, intercropping increased net economic return by $24 \%(p<0.05), 2.0 \%(p>0.05)$, and $7 \%$ $(p>0.05)$ under FMRFm/NMFp, FMFm/NMFp, and NMFm/NMFp compared to that in maize monocrop and potato monocrop, respectively. 
Table 8. Energy output and net economic return of component crops in the intercropping compared with monocrop in 2014-2015.

\begin{tabular}{|c|c|c|c|c|c|c|c|c|c|}
\hline \multirow[b]{2}{*}{ Years } & \multirow{2}{*}{$\begin{array}{c}\text { Mulch } \\
\text { Practices of } \\
\text { Maize }^{\text {a }}\end{array}$} & \multicolumn{4}{|c|}{ Energy Output (GJ ha $\mathrm{h}^{-1}$ year $^{-1}$ ) } & \multicolumn{4}{|c|}{ Net Economic Return (RMB ha-1 year $\left.^{-1}\right)^{b}$} \\
\hline & & Intercrop & $\begin{array}{c}\text { Maize } \\
\text { Monocrop }\end{array}$ & $\begin{array}{c}\text { Potato } \\
\text { Monocrop }\end{array}$ & $\Delta \mathrm{E}(\%)^{\mathrm{c}}$ & Intercrop & $\begin{array}{c}\text { Maize } \\
\text { Monocrop }\end{array}$ & $\begin{array}{c}\text { Potato } \\
\text { Monocrop }\end{array}$ & $\begin{array}{c}\Delta \text { NER } \\
(\%)^{c}\end{array}$ \\
\hline \multirow[t]{3}{*}{2014} & FMRF & $119 \mathrm{a}^{\mathrm{d}}$ & $170 \mathrm{a}$ & 52 & 7.2 & $11205 \mathrm{a}$ & $11,180 \mathrm{a}$ & 7568 & $19.5^{\sharp}$ \\
\hline & FMF & $99 \mathrm{~b}$ & $159 \mathrm{a}$ & 52 & -6.2 & $8261 \mathrm{~b}$ & 10,815 a & 7568 & $-10.1 \sharp$ \\
\hline & NMF & $80 \mathrm{c}$ & $104 \mathrm{~b}$ & 52 & 2.6 & $7978 \mathrm{~b}$ & $7335 \mathrm{~b}$ & 7568 & 7.1 \\
\hline \multirow[t]{3}{*}{2015} & FMRF & $107 \mathrm{a}$ & $164 \mathrm{a}$ & 32 & $9.2 *$ & 7584 a & $10,460 \mathrm{a}$ & 1158 & $30.6^{\sharp}$ \\
\hline & FMF & $101 \mathrm{a}$ & $156 a$ & 32 & 7.4 & $6989 a$ & $10,367 \mathrm{a}$ & 1158 & $21.3^{\sharp}$ \\
\hline & NMF & $70 \mathrm{~b}$ & $104 \mathrm{~b}$ & 32 & 2.9 & $4547 \mathrm{~b}$ & $7445 \mathrm{~b}$ & 1158 & 5.7 \\
\hline \multicolumn{10}{|l|}{ Average } \\
\hline \multirow{2}{*}{ Year (Y) } & 2014 & $99 a$ & $144 \mathrm{a}$ & $52 \mathrm{a}$ & $1.2 \mathrm{~b}$ & 9148 a & 9777 a & 7568 a & $5.5 \mathrm{~b}$ \\
\hline & 2015 & $93 a$ & $141 \mathrm{a}$ & $32 \mathrm{~b}$ & $6.5 \mathrm{a}$ & $6373 \mathrm{~b}$ & $9424 \mathrm{a}$ & $1158 \mathrm{~b}$ & $19.2 \mathrm{a}^{\sharp}$ \\
\hline \multirow{3}{*}{$\begin{array}{l}\text { Cropping } \\
\text { system } \\
\text { (C) }\end{array}$} & FMRF & $113 a$ & $167 \mathrm{a}$ & 42 & $8.1 \mathrm{a}^{*}$ & 9395 a & $10,820 \mathrm{a}$ & 4363 & $23.8 a^{\sharp}$ \\
\hline & FMF & $100 \mathrm{a}$ & $158 \mathrm{a}$ & 42 & $0.3 \mathrm{c}$ & $7625 \mathrm{~b}$ & $10,591 \mathrm{a}$ & 4363 & $2.0 \mathrm{c}$ \\
\hline & NMF & $75 \mathrm{~b}$ & $104 \mathrm{~b}$ & 42 & $2.7 \mathrm{~b}$ & $6263 c$ & $7390 \mathrm{~b}$ & 4363 & $6.6 \mathrm{~b}$ \\
\hline$Y^{\prime}$ & & ns & $\mathrm{ns}$ & $*$ & $* * *$ & $* * *$ & $\mathrm{~ns}$ & $* * *$ & $* * *$ \\
\hline $\mathrm{C}$ & & $*$ & $*$ & - & $* *$ & $* *$ & $*$ & - & $* *$ \\
\hline$Y \times C$ & & ns & ns & - & * & * & ns & - & $*$ \\
\hline
\end{tabular}

${ }^{a}$ FMRF, film mulched ridge-furrow plot; FMF, film mulched flat plot; NMF, no mulching flat plot. ${ }^{\mathrm{b}} 1 \mathrm{RMB}=6.6$ US dollar (i.e., Chinese Yuan $\mathrm{RMB}) .{ }^{c} \Delta \mathrm{E}$ and $\triangle \mathrm{NER}$ indicate energy output and net economic return increase taking into account the relative density of maize and potato in the intercrop ( $\mathrm{Pi}=0.5$ for both component crops), which is calculated as $\left[\mathrm{X}_{\text {int }}-0.5 \times\left(\mathrm{X}_{\text {mono },}, \mathrm{a}+\mathrm{X}_{\text {mono, }}, \mathrm{b}\right)\right] /\left[0.5 \times\left(\mathrm{X}_{\mathrm{mono},}, \mathrm{a}\right.\right.$ $\left.\left.+X_{\text {mono, }}, \mathrm{b}\right)\right] .{ }^{d}$ Within a column for the same year, means followed by different letters are significantly different $(p \leq 0.05)$. $\sharp$ Indicates significant differences between intercropping and monocrop taking into account the relative density of maize and potato in the intercrop. ${ }^{*}$ indicates significant differences at $p=0.05$ level; ${ }^{* *}$ indicates significant differences at $p=0.01$ level; ${ }^{* * *}$ indicates significant differences at $p=0.001$ level; ns: not significant.

\section{Discussion}

In this study, intercropping increased soil water storage deficit degree in the deeper soil layer, which means that it consumes less soil water than expected from the crop yields obtained in monocropping, which was beneficial towards promoting crop yield and water use efficiency in the semiarid environment. It is likely because the intensified soil water competition in intercropping forced maize to extract more water from deeper soil layers.

Intercropping treatments of FMFm/NMFp and NMFm/NMFp increased water use compared to maize monocrop. However, the plastic film mulched ridge-furrow system had great advantages in terms of promoting water infiltration, reducing soil evaporation, and increasing soil water storage [48], which timely replenished the soil moisture and reduced the depletion of soil water. Potato consumed greater soil water than maize because of the slower early growth of potato and relatively small canopy which did not timely cover soil causing high soil evaporation, thereby increasing water consumption [31]. Adding potato crop into intercropping without mulching inevitably depleted soil water which in the long run might cause severe soil dryness and limit yield and WUE $[8,40]$. However, if the soil management practices are appropriate, intercropping of film mulching and ridge-furrow sowing of maize with potato (FMRFm/NMFp) did not increase water consumption.

Intercropping had increased LER and WER, especially for FMRFm/NMFp, which is consistent with the previous studies $[5,7,37,38]$. The mechanisms for high LER and WER in the maize/potato intercropping might be: (1) complementarity for water use between maize and potato due to the difference in root distribution of the component crops along with soil layer [37,38,49], for example, the roots of potato mainly distributes 0-40 cm soil layer [50], but the root system of maize can reach to $100 \mathrm{~cm}$ soil depth and could extract stored soil water from deeper soil layers [38], and complementarity for a light interception as a result of increased crop canopy architecture under the relatively low planting density in intercropping, leading to increased production of dry matter, thereby increased yields [7,17]; (2) potato is a shade loving crop [51-53], and thus aboveground shading from intercropped maize might be beneficial for the growth and yield. These 
changes under intercropping were beneficial towards promoting land productivity and water use efficiency when compared to monocropping.

However, in contrast to our expectation, the partial LER of maize in intercropping was close to half the yield of maize in monocropping. These results suggest that intercropped maize did not achieve higher yield and water use efficiency when compared to monocropping of maize, although some studies claimed that intercropping increased maize yield and WUE under irrigation $[5,7,54]$. This discrepancy may be associated with differences in planting densities since high planting density $\left(90,000-100,000\right.$ plants ha $\left.^{-1}\right)$ and water supply was used in these studies. Especially under high planting density, increased the border-row proportion in intercropping increased crop yield and WUE through the increases in crop canopy growth and light interception due to a relatively low density $[5,55]$. On the contrary, in this study, high water consumption of potato in intercropping would intensify the water competition between plants of potato and maize, thereby limiting the increase in maize yield. This can be explained by the greater LER and WER of potatoes than those of maize.

Maize yield was higher under plastic mulching than without mulching, since plastic film mulching greatly increased the soil temperature and moisture and reduced soil evaporation $[20,48]$, which subsequently enhanced crop transpiration and increased crop yield [11,56-59]. FMRFm/NMFp had the greatest LER and WER, mainly because the increased soil water infiltration and storage under the FMRF system enhanced maize and potato yield, as has been shown in the previous studies $[18,20,22,48]$. In this study, intercropping increased energy output and net economic return as a result of high LER, but the increases in energy output and net economic return were only significantly greater under FMRFm/NMFp, suggesting that FMRFm/NMFp is the optimal intercropping system on the western Loess Plateau.

\section{Conclusions}

Adding a high-water consumption crop (i.e., potato) into an ecosystem inevitably depleted soil water; therefore, in the long run, intercropping treatments of film mulched or no mulched flat planting of maize along with flat planting of potato might cause severe soil dryness and reduced yield and WUE. However, the intercropping system of plastic film fully mulched ridge-furrow maize and no-mulching flat planting of potato (FMRFm/NMFp) relatively consumed less water while producing higher yield, water use efficiency, energy output, and net economic return without increasing soil water depletion. Considering that the test has only been carried out at one site for two years, the findings of our study may have uncertainty. Before widely applying this conclusion, more experimental studies at other sites in the Western Loess Plateau should be encouraged to evaluate the trending developments.

Author Contributions: Conceptualization: L.L.; methodology: L.W., J.X. and Z.L.; data analysis: J.X.; writing — original draft preparation: J.X., L.W.; writing—review and editing: J.X., L.W., L.L., S.A., E.Z. and S.K.F.; supervision: L.L. and L.W.; funding acquisition: L.L.; project administration: J.X. All authors have read and agreed to the published version of the manuscript.

Funding: This research was funded by the Education science and technology innovation project of Gansu Province (GSSYLXM-02), the National Natural Science Foundation of China (42167008 and 31761143004), the Natural Science Foundation of Gansu Province (21ZD4NF043), the Sheng Tongsheng innovation funds of Gansu Agricultural University (GSAU-STS-2021-21), the Fuxi young funds of Gansu Agricultural University (GAUfx-04Y09), and the Young Instructor Fund Project of Gansu Agricultural University (GAU-QDFC2020-03).

Institutional Review Board Statement: Not applicable.

Informed Consent Statement: Not applicable.

Data Availability Statement: Not applicable. 
Acknowledgments: We appreciate the excellent technical assistance for field sampling and laboratory tests provided by undergraduate and graduate students at the Gansu Agricultural University Rainfed Agricultural Experimental Station.

Conflicts of Interest: The authors declare no conflict of interest.

\section{References}

1. Raseduzzaman, M.; Jensen, E.S. Does intercropping enhance yield stability in arable crop production? A meta-analysis. Eur. J. Agron. 2017, 91, 25-33. [CrossRef]

2. Mueller, N.D.; Gerber, J.S.; Johnston, M.; Ray, D.K.; Ramankutty, N.; Foley, J.A. Closing yield gaps through nutrient and water management. Nature 2012, 490, 254-257. [CrossRef]

3. Xue, J.F.; Liu, S.L.; Chen, Z.D.; Chen, F.; Lal, R.; Tang, H.M. Assessment of carbon sustainability under different tillage systems in a double rice cropping system in Southern China. Int. J. Life Cycle Assess. 2014, 19, 1581-1592. [CrossRef]

4. Yang, X.; Gao, W.; Zhang, M.; Chen, Y.; Sui, P. Reducing agricultural carbon footprint through diversified crop rotation systems in the North China Plain. J. Clean. Prod. 2014, 76, 131-139. [CrossRef]

5. Fan, Z.; Chai, Q.; Yu, A.; Zhao, C.; Yin, W.; Hu, F. Water and radiation use in maize/pea intercropping is enhanced with increased plant density. Agron. J. 2020, 112, 1-12. [CrossRef]

6. Renard, D.; Tilman, D. National food production stabilized by crop diversity. Nature 2019, 571, 257-260. [CrossRef]

7. Zhao, C.; Fan, Z.; Coulter, J.A.; Yin, W.; Hu, F.; Yu, A. High Maize Density Alleviates the Inhibitory Effect of Soil Nitrogen on Intercropped Pea. Agronomy 2020, 10, 248. [CrossRef]

8. Chai, Q.; Gan, Y.; Turner, N.C.; Zhang, R.Z.; Yang, C.; Niu, Y. Water-saving innovations in Chinese agriculture. Adv. Agron. 2014, 126, 149-202.

9. Mitchell, J.P.; Singh, P.N.; Wallender, W.W.; Munk, D.S.; Wroble, J.F.; Horwath, W.R. No-tillage and high-residue practices reduce soil water evaporation. Calif. Agric. 2012, 66, 55-61. [CrossRef]

10. Peng, Z.; Wang, L.; Xie, J.; Li, L.; Coulter, J.A.; Zhang, R. Conservation Tillage Increases Water Use Efficiency of Spring Wheat by Optimizing Water Transfer in a Semi-Arid Environment. Agronomy 2019, 9, 583. [CrossRef]

11. Peng, Z.; Wang, L.; Xie, J.; Li, L.; Coulter, J.A.; Zhang, R. Conservation tillage increases yield and precipitation use efficiency of wheat on the semi-arid Loess Plateau of China. Agric. Water Manag. 2020, 231, 106024. [CrossRef]

12. Deng, X.; Shan, L.; Zhang, H.; Turner, N.C. Improving agricultural water use efficiency in arid and semiarid areas of China. Agric. Water Manag. 2006, 80, 23-40. [CrossRef]

13. Wang, L.; Palta, J.A.; Chen, W.; Chen, Y.; Deng, X. Nitrogen fertilization improved water-use efficiency of winter wheat through increasing water use during vegetative rather than grain filling. Agric. Water Manag. 2018, 197, 41-53. [CrossRef]

14. Sun, M.; Gao, Z.; Zhao, W.; Deng, L.; Deng, Y.; Zhao, H. Effect of subsoiling in fallow period on soil water storage and grain protein accumulation of dryland wheat and its regulatory effect by nitrogen application. PLoS ONE 2013, 8, e75191. [CrossRef] [PubMed]

15. Wang, J.; Liu, W.; Dang, T. Responses of soil water balance and precipitation storage efficiency to increased fertilizer application in winter wheat. Plant Soil 2011, 347, 41-51. [CrossRef]

16. Wang, L.; Li, Q.; Coulter, J.A.; Xie, J.; Luo, Z.; Zhang, R. Winter wheat yield and water use efficiency response to organic fertilization in northern China: A meta-analysis. Agric. Water Manag. 2020, 229, 105934. [CrossRef]

17. Fan, T.; Stewart, B.A.; Yong, W.; Junjie, L.; Guangye, Z. Long-term fertilization effects on grain yield, water-use efficiency and soil fertility in the dryland of Loess Plateau in China. Agric. Ecosyst. Environ. 2005, 106, 313-329. [CrossRef]

18. Xie, J.; Wang, L.; Li, L.; Coulter, J.A.; Chai, Q.; Zhang, R. Subsoiling increases grain yield, water use efficiency, and economic return of maize under a fully mulched ridge-furrow system in a semiarid environment in China. Soil Tillage Res. 2020, $199,104584$. [CrossRef]

19. Zhang, S.; Li, P.; Yang, X.; Wang, Z.; Chen, X. Effects of tillage and plastic mulch on soil water, growth and yield of spring-sown maize. Soil Tillage Res. 2011, 112, 92-97. [CrossRef]

20. Gan, Y.; Siddique, K.H.; Turner, N.C.; Li, X.G.; Niu, J.Y.; Yang, C. Ridge-furrow mulching systems-An innovative technique for boosting crop productivity in semiarid rain-fed environments. In Advances in Agronomy; Elsevier: Amsterdam, The Netherlands, 2013; Volume 118, pp. 429-476.

21. Li, F.; Li, X.; Javaid, M.; Ashraf, M.; Zhang, F. Ridge-furrow plastic film mulching farming for sustainable dryland agriculture on the Chinese loess plateau. Agron. J. 2020, 112, 3284-3294. [CrossRef]

22. Zhou, L.; Jin, S.; Liu, C.; Xiong, Y.; Si, J.; Li, X. Ridge-furrow and plastic-mulching tillage enhances maize-soil interactions: Opportunities and challenges in a semiarid agroecosystem. Field Crop. Res. 2012, 126, 181-188. [CrossRef]

23. Wang, Z.; Zhang, H.; Lu, X.; Wang, M.; Chu, Q.; Wen, X. Lowering carbon footprint of winter wheat by improving management practices in North China Plain. J. Clean. Prod. 2016, 112, 149-157. [CrossRef]

24. Xue, X.; Landis, A.E. Eutrophication Potential of Food Consumption Patterns. Environ. Sci. Technol. 2010, 44, 6450-6456. [CrossRef]

25. Zhang, D.; Shen, J.; Zhang, F.; Li, Y.; Zhang, W. Carbon footprint of grain production in China. Sci. Rep. 2017, 7, 4126. [CrossRef] [PubMed] 
26. He, G.; Wang, Z.; Li, S.; Malhi, S. Plastic mulch: Tradeoffs between productivity and greenhouse gas emissions. J. Clean. Prod. 2018, 172, 1311-1318. [CrossRef]

27. Wang, L.; Coulter, J.A.; Li, L.; Luo, Z.; Xie, J. Plastic mulching reduces nitrogen footprint of food crops in China: A meta-analysis. Sci. Total. Environ. 2020, 748, 141479. [CrossRef]

28. Yan, C.; He, W.; Turner, N. Plastic-film mulch in Chinese agriculture: Importance and problems. World Agric. 2014, 4, 32-36.

29. Gao, H.; Yan, C.; Liu, Q.; Ding, W.; Chen, B.; Li, Z. Effects of plastic mulching and plastic residue on agricultural production: A meta-analysis. Sci. Total. Environ. 2018, 651, 484-492. [CrossRef]

30. Liu, C.; Jin, S.; Zhou, L.; Jia, Y.; Li, F.; Xiong, Y. Effects of plastic film mulch and tillage on maize productivity and soil parameters. Eur. J. Agron. 2009, 31, 241-249. [CrossRef]

31. Xie, J.; Chai, Q.; Zhang, R.; Luo, Z.; Li, L.; Niu, Y. Suitable Succession Crop Screening After Perennial Alfalfa for Soil Dedication Restoration in Loess Plateau. J. Soil Water Conserv. 2014, 5, 51-57.

32. Qin, S.; Yeboah, S.; Wang, D.; Zhang, J.; Unc, A. Effects of ridge-furrow and plastic mulching planting patterns on microflora and potato tuber yield in continuous cropping soil. Soil Use Manag. 2016, 32, 465-473. [CrossRef]

33. Khan, S.; Shah, A.; Nawaz, M.; Khan, M. Impact of different tillage practices on soil physical properties, nitrate leaching and yield attributes of maize (Zea mays, L.). J. Soil Sci. Plant Nutr. 2017, 17, 1-12. [CrossRef]

34. Lu, F.; Wang, X.; Han, B.; Ouyang, Z.; Duan, X.; Zheng, H. Soil carbon sequestrations by nitrogen fertilizer application, straw return and no-tillage in China's cropland. Glob. Chang. Biol. 2009, 15, 281-305. [CrossRef]

35. Buragienè, S.; Šarauskis, E.; Romaneckas, K.; Sasnauskienè, J.; Masilionytè, L.; Kriaučiūnienè, Z. Experimental analysis of CO 2 emissions from agricultural soils subjected to five different tillage systems in Lithuania. Sci. Total. Environ. 2015, 514, 1-9. [CrossRef]

36. Li, L.; Huang, G.; Zhang, R.; Jin, X.; Chan, K. Effects of conservation tillage on soil water regimes in rainfed areas. Acta Ecol. Sin. 2005, 25, 2326-2332.

37. Ren, J.; Zhang, L.; Duan, Y.; Zhang, J.; Evers, J.; Zhang, Y. Intercropping potato (Solanum tuberosum, L.) with hairy vetch (Vicia villosa) increases water use efficiency in dry conditions. Field Crop. Res. 2019, 240, 168-176. [CrossRef]

38. Ren, Y.; Wang, X.; Zhang, S.; Palta, J.A.; Chen, Y. Influence of spatial arrangement in maize-soybean intercropping on root growth and water use efficiency. Plant Soil 2017, 415, 131-144. [CrossRef]

39. Li, C.; Hoffland, E.; Kuyper, T.W.; Yu, Y.; Zhang, C.; Li, H. Syndromes of production in intercropping impact yield gains. Nat. Plants 2020, 6, 653-660. [CrossRef] [PubMed]

40. Li, L. Intercropping enhances agroecosystem services and functioning: Current knowledge and perspectives. Chin. J. Eco-Agric. 2016, 24, 403-415.

41. Qiang, C. Research Progress on Mechanism of High Efficient Water Utilization in Intercropping System. J. Agric. Sci. Technol. 2008, 10, 11-15.

42. Midmore, D.J. Agronomic modification of resource use and intercrop productivity. Field Crop. Res. 1993, 34, 357-380. [CrossRef]

43. Garrity RAMP. Resource capture and utilization in intercropping: Water. Field Crop. Res. 1993, 3, $303-317$.

44. Fang, Y.; Miao, Q.; Liu, S.; Xu, B.; Chen, Y. Optimal Wheat Seeding Rate is Influenced by Cultivar-Specific Topsoil and Subsoil Root Traits. Agron. J. 2019, 111, 3150-3160. [CrossRef]

45. Gong, Z. Chinese Soil Taxonomy; China Science Press: Beijing, China, 2011; pp. 5-215. (In Chinese)

46. Mead, R.; Willey, R.W. The Concept of a 'Land Equivalent Ratio' and Advantages in Yields from Intercropping. Exp. Agric. 1980, 16, 217-228. [CrossRef]

47. Liu, H.; Wang, Z.; Yu, R.; Li, F.; Li, K.; Cao, H. Optimal nitrogen input for higher efficiency and lower environmental impacts of winter wheat production in China. Agric. Ecosyst. Environ. 2016, 224, 1-11. [CrossRef]

48. Jiang, X.; Li, X.G. Assessing the effects of plastic film fully mulched ridge-furrow on rainwater distribution in soil using dye tracer and simulated rainfall. Soil Tillage Res. 2015, 152, 67-73. [CrossRef]

49. Bai, W.; Sun, Z.; Zheng, J.; Du, G.; Feng, L.; Cai, Q. Mixing trees and crops increases land and water use efficiencies in a semi-arid area. Agric. Water Manag. 2016, 178, 281-290. [CrossRef]

50. Zin El-Abedin, T.K.; Mattar, M.A.; Alazba, A.A.; Al-Ghobari, H.M. Comparative effects of two water-saving irrigation techniques on soil water status, yield, and water use efficiency in potato. Sci. Hortic. 2017, 225, 525-532. [CrossRef]

51. Li, Q.; Li, H.; Zhang, L.; Zhang, S.; Chen, Y. Mulching improves yield and water-use efficiency of potato cropping in China: A meta-analysis. Field Crop. Res. 2018, 221, 50-60. [CrossRef]

52. Li, Y.; Cheng, P.; Xiong, X.; Hong, Y. DNA Methylation in Potato Under Drought Stress. Chin. Potato J. 2012, 26, 11-15.

53. Wang, L.; Coulter, J.A.; Palta, J.A.; Xie, J.; Deng, X. Mulching-Induced Changes in Tuber Yield and Nitrogen Use Efficiency in Potato in China: A Meta-Analysis. Agronomie 2019, 9, 793. [CrossRef]

54. Yin, W.; Fan, Z.; Hu, F.; Yu, A.; Zhao, C.; Chai, Q. Innovation in alternate mulch with straw and plastic management bolsters yield and water use efficiency in wheat-maize intercropping in arid conditions. Sci. Rep. 2019, 9, 1-14. [CrossRef] [PubMed]

55. Wang, R.; Sun, Z.; Zhang, L.; Yang, N.; Feng, L.; Bai, W. Border-row proportion determines strength of interspecific interactions and crop yields in maize/peanut strip intercropping. Field Crop. Res. 2020, 253, 107819. [CrossRef]

56. Yu, Y.; Turner, N.C.; Gong, Y.; Li, F.; Fang, C.; Ge, L. Benefits and limitations to straw-and plastic-film mulch on maize yield and water use efficiency: A meta-analysis across hydrothermal gradients. Eur. J. Agron. 2018, 99, 138-147. [CrossRef] 
57. Zheng, J.; Fan, J.; Zou, Y.; Chau, H.; Zhang, F. Ridge-furrow plastic mulching with a suitable planting density enhances rainwater productivity, grain yield and economic benefit of rainfed maize. J. Arid. Land 2020, 12, 181-198. [CrossRef]

58. Zhang, X.; Yang, L.; Xue, X.; Kamran, M.; Ahmad, I.; Dong, Z. Plastic film mulching stimulates soil wet-dry alternation and stomatal behavior to improve maize yield and resource use efficiency in a semi-arid region. Field Crop. Res. 2019, 233, 101-113. [CrossRef]

59. Zhang, X.; Zhao, J.; Yang, L.; Kamran, M.; Xue, X.; Dong, Z. Ridge-furrow mulching system regulates diurnal temperature amplitude and wetting-drying alternation behavior in soil to promote maize growth and water use in a semiarid region. Field Crop. Res. 2019, 233, 121-130. [CrossRef] 